\title{
The translationally-invariant coupled cluster method in coordinate space
}

\author{
I. Moliner a,1, R.F. Bishop ${ }^{\text {a }}$, N.R. Walet ${ }^{\text {a }}$, R. Guardiola ${ }^{\text {b }}$ J. Navarro ${ }^{\text {c }}$, M. Portesi ${ }^{\text {d }}$ \\ ${ }^{\mathrm{a}}$ Dept. of Physics, UMIST, P.O. Box 88, Manchester M60 1QD, UK \\ b Dpto. de Física Atómica, Molecular y Nuclear, Universidad de Valencia, Avda. Dr. Moliner 50, E-46100 Burjassot, Spain \\ ${ }^{\mathrm{c}}$ IFIC, Edificio Institutos Paterna, Apdo. 2085, E-46071 Valencia, Spain \\ ${ }^{\mathrm{d}}$ Dpto. de Física, UNLP, C.C. 67, (1900) La Plata, Argentina
}

Received 10 November 1999; accepted 13 March 2000

Editor: J.-P. Blaizot

\begin{abstract}
We study a formulation of the translationally-invariant coupled cluster method in coordinate space. Previous calculations in configuration space showed poor convergence, a problem that the new formulation is expected to remedy. This question is investigated for a system of bosons interacting through the Wigner part of the Afnan-Tang S3 interaction, where previous results exist. (C) 2000 Elsevier Science B.V. All rights reserved.
\end{abstract}

Keywords: Many-body techniques; Two-body correlations; Coupled cluster methods

The coupled cluster method (CCM) provides a well studied formalism to describe many-body systems in many areas of physics. We have been studying the application of this method to the nuclear problem [1,2]. In our work we have emphasised the importance of a correct treatment of the centre-ofmass motion. This requires a modification to the CCM formalism, which has mainly been studied up till now at the level of two-body correlations. The resulting theory (TICC2) has been investigated and formulated in configuration space $[3,4]$, and was applied mainly to bosonic systems, where it was found to suffer from very slow convergence with

\footnotetext{
${ }^{1}$ Phone: +44 (0)161 200 8704; fax: +44 (0)161 200 4303; e-mail: moliner@dirac.phy.umist.ac.uk
}

respect to the size of the single-particle space. It was then realized that a linearized version of the theory (TICI2) can easily be reformulated in coordinate space, avoiding these convergence problems. In this letter we shall describe a comparable formulation of TICC2 in coordinate space and compare it with previous results in configuration space for model systems of bosonic nucleons interacting through a Wigner-type potential.

In general, the CCM ansatz for the wave function of an $A$-particle system is generated by the exponential form

$|\Psi\rangle=\exp (\hat{S})|\Phi\rangle$

where $\hat{S}$ is a sum of one-body, two-body, etc., operators acting on an uncorrelated reference state 
$|\Phi\rangle$. It has been shown [3] that when the operator $\hat{S}$ is restricted to one- and two-body correlations, the translationally invariant form can be represented in coordinate space in terms of a two-body function $f\left(r_{i j}\right)$, depending only on the relative coordinate of a pair. Moreover, the uncorrelated reference state must be a product of harmonic oscillator (HO) single particle wave functions to remove the center-of-mass motion properly. Therefore, in coordinate space, the TICC2 wave function can be written as $[5,6]$

$$
\begin{aligned}
& \Psi\left(\boldsymbol{r}_{1}, \ldots, \boldsymbol{r}_{A}\right) \\
& =\left(1+\sum_{i<j} f\left(r_{i j}\right)+\frac{1}{2 !} \sum_{i<j} f\left(r_{i j}\right) \sum_{k<l}^{\prime} f\left(r_{k l}\right)\right. \\
& \quad+\frac{1}{3 !} \sum_{i<j} f\left(r_{i j}\right) \sum_{k<l}^{\prime} f\left(r_{k l}\right) \sum_{m<n}^{\prime \prime} f\left(r_{m n}\right) \\
& \quad+\ldots) \Phi\left(\boldsymbol{r}_{1}, \ldots, \boldsymbol{r}_{A}\right),
\end{aligned}
$$

where $\Phi\left(\boldsymbol{r}_{1}, \ldots, \boldsymbol{r}_{A}\right)$ is an uncorrelated $\mathrm{HO}$ wave function; and the primed sums denote that all the indices in the nested sums are distinct, showing explicitly the characteristic CCM property of dealing only with independent excitations. It is worth noting that this wave function coincides with the independent pair approximation to a Jastrow wave function, proposed some years ago [7]. If we only take up to linear terms in $f$ in the wave function of Eq. (2), we obtain the one used in the pairwise translationally-invariant configuration-interaction (TICI2) method.

The problem is thus reduced to a determination of the pair correlation function $f(r)$. The intermediate normalization condition $\langle\Phi \mid \Psi\rangle=1$, inherent to CCM, implies that the correlation function must be restricted by the relation

$$
\begin{aligned}
& \langle\Phi|\left(\sum_{i<j} f\left(r_{i j}\right)+\frac{1}{2 !} \sum_{i<j} f\left(r_{i j}\right) \sum_{k<l}{ }^{\prime} f\left(r_{k l}\right)+\ldots\right) \\
& \quad \times|\Phi\rangle=0 .
\end{aligned}
$$

In the case of a bosonic system where $\Phi$ is a simple boson condensate, this condition is equivalent to

$$
\left\langle\phi_{000}\left(\boldsymbol{r}_{1}\right) \phi_{000}\left(\boldsymbol{r}_{2}\right)\left|f\left(r_{12}\right)\right| \phi_{000}\left(\boldsymbol{r}_{1}\right) \phi_{000}\left(\boldsymbol{r}_{2}\right)\right\rangle=0 \text {, }
$$

where $\phi_{000}(\boldsymbol{r})$ is the $0 s \mathrm{HO}$ single particle wave function, in the usual $\phi_{n l m}$ basis.
Following the standard approach to the CCM [8], we obtain equations for $f$ and the ground-state energy by projecting the Schrödinger equation both onto the uncorrelated state, and onto a set of generic excited states containing up to two-body correlations. These states are obtained by multiplying the uncorrelated state by $\sum_{i<j} g\left(r_{i j}\right)$, where $g$ is an arbitrary function that satisfies the condition of Eq. (4). We then obtain an integro-differential equation from which one can determine the correlation function $f$, plus a supplementary expression for the ground-state energy.

We work with a standard two-body hamiltonian, comprising sums of single-body kinetic energy terms and translationally-invariant two-body potential terms. In that case the equations can be most succinctly presented using a diagrammatic notation [6], where a circle represents a particle, solid lines denote the correlation function $f$, dashed lines stand for the interaction, crosses indicate the action of the kinetic energy operator, and wavy lines denote the function $g$. The equation corresponding to the projection onto a generic excitation takes the form

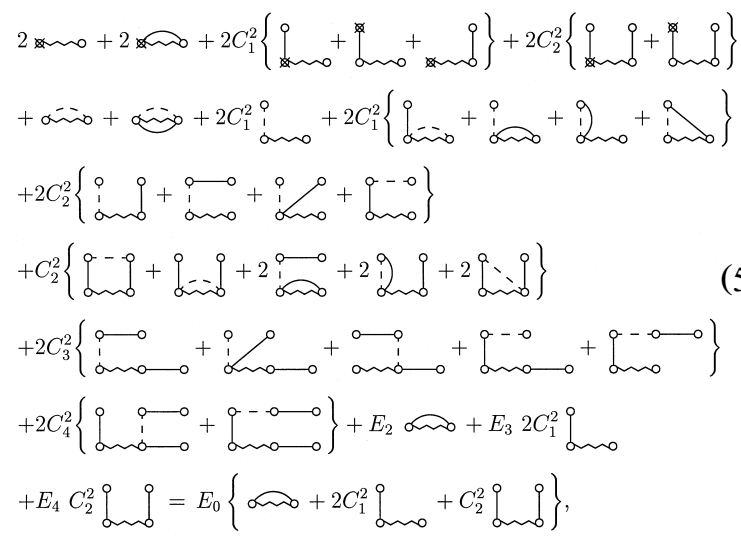

where $C_{k}^{n} \equiv(A-n)(A-n-1) \ldots(A-n-k+1)$ are statistical factors counting the different equivalent labellings of each diagram. The quantities $E_{n}$ appearing in Eq. (5) are given by

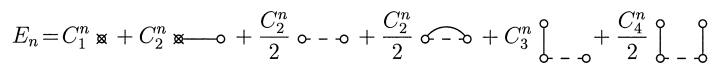

and they are related to unlinked diagrams. Most importantly, $E_{0}$ corresponds to the ground-state energy. 
To clarify the meaning of the diagrammatic notation consider the following diagram,

and $=\int \Phi^{*} g^{*}\left(r_{12}\right) V\left(r_{34}\right) f\left(r_{13}\right) f\left(r_{24}\right) \Phi d \mathbf{r}_{1} \ldots d \mathbf{r}_{A}$.

From this expression we see that Eq. (5) is the integral of the function $g^{*}\left(r_{12}\right)$ times a non-linear functional, depending on the pair correlation $f(r)$, equated to zero,

$\int g^{*}\left(r_{12}\right) F[f] d \boldsymbol{r}_{1} \ldots d \boldsymbol{r}_{A}=0$.

Given that $g(r)$ is not a fully arbitrary function, because of the required orthogonality of the excited states with respect to the reference state, one cannot straightforwardly obtain an integro-differential equation for $f(r)$. We could introduce a Lagrange multiplier, but it is easier to introduce a completely arbitrary function $G(r)$, and replace $g$ by $G-\langle\Phi|G| \Phi\rangle$ in Eq. (5), which obviously satisfies the constraint. After this substitution, the integro-differential equation for $f$ is easily obtained. Moreover, we can avoid the cumbersome non-linear integro-differential equation by expanding both $G$ and $f$ in a suitable basis, leading to a system of algebraic non-linear coupled equations. We have chosen a set of gaussians as our (overcomplete) basis. Our previous experience with the linear TICI2 formalism [1,2] has shown this method to be both accurate and economical.

In Table 1 we list results for different systems of nucleons considered as bosons and interacting through the Wigner part of the Afnan-Tang S3 interaction [9]. There is excellent agreement between the HO configuration-space calculations [4] and the pre-

Table 1

Binding energies (in $\mathrm{MeV}$ ) for various "bosonic nuclei" with the Wigner part of the S3 interaction and for the different orders of truncation of the equations in powers of $f$. Order 1 corresponds to the TICI 2 case, and Order 4 is the full TICC2 result. The last row shows the full TICC2 configuration-space results [4].

\begin{tabular}{lllll}
\hline & $A=4$ & $A=8$ & $A=16$ & $A=40$ \\
\hline Order 1 & 25.42 & 225.46 & 1131.2 & 7495.3 \\
Order 2 & 25.60 & 235.12 & 1235.7 & 8457.1 \\
Order 3 & & 235.12 & 1235.1 & 8458.5 \\
Order 4 & & 235.12 & 1235.1 & 8458.7 \\
Configuration & 25.49 & 235.03 & 1234.9 & 8456.6 \\
\hline
\end{tabular}

sent coordinate-space ones. It is worth noting that the coordinate-space numbers are fully converged, with the use of between 10 and 14 gaussians. By contrast, in HO configuration space we used up to 30 amplitudes (corresponding to single-particle excitation energies up to $60 \hbar \omega$ ), and still not reaching full convergence in some cases. Moreover, working in coordinate-space results in a much faster computation of the required expansion coefficients. For these bosonic systems the computer time needed is roughly 500 times smaller as compared with the calculations in configuration space.

Another interesting feature of the method is revealed when we compare the different results obtained as a function of a truncation of the equations in powers of $f$. In this truncation, the first order corresponds to the linear approach (TICI2) and the fourth order to the full TICC2 approach. Although in the light $A=4$ system the contribution of non-linear terms is not very relevant, for the heavier systems the improvement in the energy is indeed quite remarkable, as was already noticed in configurationspace calculations [4]. It is interesting to note that the third- and fourth-order terms give an almost negligible contribution, so that one may safely simplify Eq. (5) by keeping only up to quadratic terms in $f(r)$.

To obtain optimal results at this level of approximation we should also have adjusted $\alpha$, the HO inverse length parameter. In order to compare with the configuration-space results, the calculations in coordinate-space were performed using the same values for $\alpha$. In the configuration-space case, which is extremely time-consuming, this parameter was fixed to give the minimum energy in the linear approximation, which is relatively quickly computed. Whether this is the best choice for the full calculation was unclear. We have checked this assumption using the current approach, and it turns out that it is essentially the correct choice in the lighter systems, but not in the heavier ones. In the $A=16$ system the minimum is in fact $1237.2 \mathrm{MeV}$ (for $\alpha=0.78 \mathrm{fm}^{-1}$ ), instead of $1235.1 \mathrm{MeV}$ (for $\alpha=0.81 \mathrm{fm}^{-1}$ ). In the $A=40$ case the displacement is greater, with a result of 8499.2 $\mathrm{MeV}$ (for $\alpha=0.78 \mathrm{fm}^{-1}$ ) to be compared with $8458.7 \mathrm{MeV}$ (for $\alpha=0.83 \mathrm{fm}^{-1}$ ).

In this work we have investigated a new formulation of the TICC2 method in coordinate space as a first step towards its application to nuclear systems. 
We have seen that with the coordinate-space formulation we can overcome the convergence problems present in the HO configuration space, with a remarkable saving of computer time as well. At the same time, we have seen that most relevant contributions to the energy are already included in the second order. This observation is likely to be helpful in fermionic systems, where we need to tackle the additional complications arising from the Fermi statistics. The implementation of the TICC2 equations for fermionic systems is the natural next step in this investigation, and it is already under study.

\section{Acknowledgements}

This work was supported by a research grant (GR/L22331) from the Engineering and Physical Sciences Research Council (EPSRC) of Great Britain and by DGICyT (Spain), grant PB97-1139. M.P. acknowledges CONICET (Argentina).

\section{References}

[1] R. Guardiola, P.I. Moliner, J. Navarro, R.F. Bishop, A. Puente, N.R. Walet, Nucl. Phys. A 609 (1996) 218.

[2] R.F. Bishop, R. Guardiola, I. Moliner, J. Navarro, M. Portesi, A. Puente, N.R. Walet, Nucl. Phys. A 643 (1998) 243.

[3] R.F. Bishop, M.F. Flynn, M.C. Boscá, E. Buendía, R. Guardiola, Phys. Rev. C 42 (1990) 1341.

[4] R. Guardiola, I. Moliner, J. Navarro, M. Portesi, Nucl. Phys. A 628 (1998) 187.

[5] R. Guardiola, I. Moliner, J. Navarro, M. Portesi, in: J.M. Arias, M.I. Gallardo, M. Lozano (Eds.), Many-Body Theory of Correlated Fermion Systems, World Scientific, 1998, p. 25.

[6] J. Navarro, R. Guardiola, M. Portesi, I. Moliner, in: R.F. Bishop, N.R. Walet (Eds.), Microscopic Approaches to the Structure of Light Nuclei Advances in Quantum Many-Body Theory, vol. 2, World Scientific, Singapore 2000, in preparation.

[7] J.C. Owen, Ann. Phys. (NY) 118 (1979) 373.

[8] H. Kümmel, K.H. Lührmann, J.G. Zabolitzky, Phys. Rep. 36C (1978) 1.

[9] I.R. Afnan, Y.C. Tang, Phys. Rev. 175 (1968) 1337. 